\title{
Problèmes liés à l'accroissement de la productivité chez la lapine reproductrice
}

\section{1 / Quelques données zootechniques}

La lapine reproductrice est élevée aujourd'hui principalement en cages individuelles grillagées dans des salles éclairées 16 heures par jour. Le lapin est exploité pour sa chair, mais aussi pour son poil (angora) et sa fourrure ; c'est aussi un animal de laboratoire et parfois un animal de compagnie. Les lapins producteurs de chair sont de format moyen (4 kg pour les populations "femelle ", essentiellement croisées) ; les populations " mâle " sont parfois plus lourdes (4 à $5,5 \mathrm{~kg}$ selon les souches). C'est une espèce polytoque à ovulation provoquée par le coït ; une lapine peut entreprendre une gestation dès la fin de la précédente. Elle peut donc être à la fois gestante et allaitante. La vie reproductive commence vers l'âge de 16 à 18 semaines. La durée de gestation est de 31 jours et le sevrage est pratiqué couramment à 28 jours, et de plus en plus fréquemment à 35 jours. En théorie, une lapine peut donc faire près de 12 mise bas par an. En pratique, le mode de conduite le plus fréquent est la saillie 10 à 12 jours après la mise bas, avec donc un intervalle théorique entre mise bas de 42 jours (dont environ la moitié de cette période simultanément en gestation et lactation). Ceci permet des conduites en bandes avec différents systèmes (bandes tous les 21 jours avec passage des femelles d'une bande à l'autre ou bande unique tous les 42 jours). Un peu moins de $20 \%$ des femelles sont éliminées sans pro- duire. Les autres assurent une carrière qui peut atteindre 16 mise bas en deux ans. La " fonte de cheptel ", c'est-à-dire le taux de disparition des femelles, est de l'ordre de 5 à 10 $\%$ par mois. Les lapereaux pèsent environ 600 $\mathrm{g}$ au sevrage ; ils sont abattus au poids de 2 $350 \mathrm{~g}$ en moyenne, à l'âge de 9 à 11 semaines.

La figure 1 présente l'évolution des principales composantes de la productivité dans les troupeaux suivis en gestion technique sur les 14 dernières années (Kœhl 1997). On constate que tous ces paramètres ont connu une amélioration sensible, malgré une tendance à la diminution du rythme de reproduction, en raison de l'évolution récente du mode de conduite des troupeaux. Est-ce à dire qu'il n'y a aucun problème lié à l'augmentation de la productivité chez la lapine?

\section{2 / Poids des lapereaux à la naissance et au sevrage}

\section{1 / Effet de la sélection pour la prolificité}

Une expérience de sélection pour la taille de la portée a été menée par l'INRA sur deux souches de lapins pendant 18 générations. Dans la souche A1077, un progrès génétique de 0,07 lapereau sevré par portée et par génération a été réalisé. Le poids total de la portée
INRA Station

d'Amélioration génétique

des Animaux,

BP 27,

31326 Castanet-

Tolosan

Figure 1. Evolution de quelques paramètres de productivité de 1983 à 1996 (base 100 en 1983). Les valeurs pour l'année 1996 figurent entre parenthèses dans la légende.

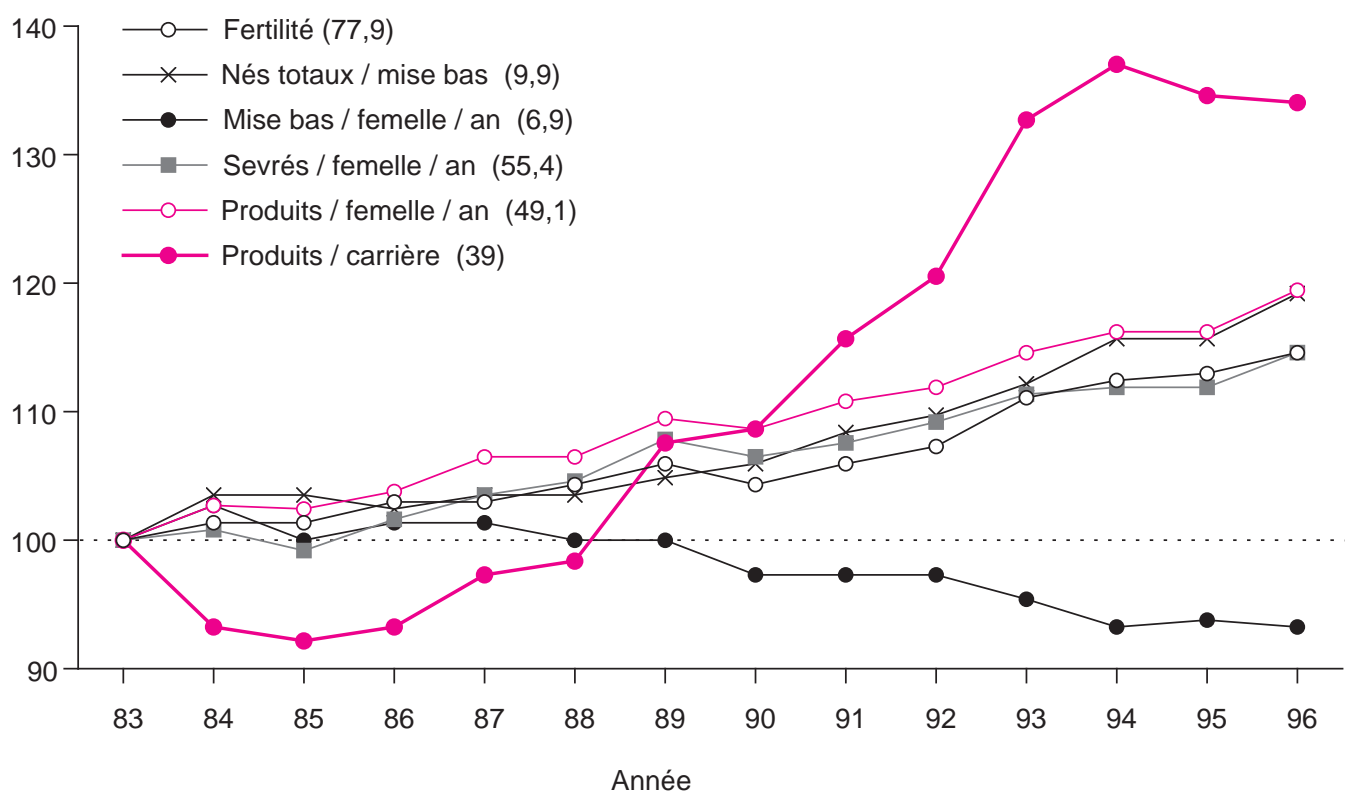


Figure 2. Evolution des valeurs génétiques (en unités d'écart type) dans la souche A1077.

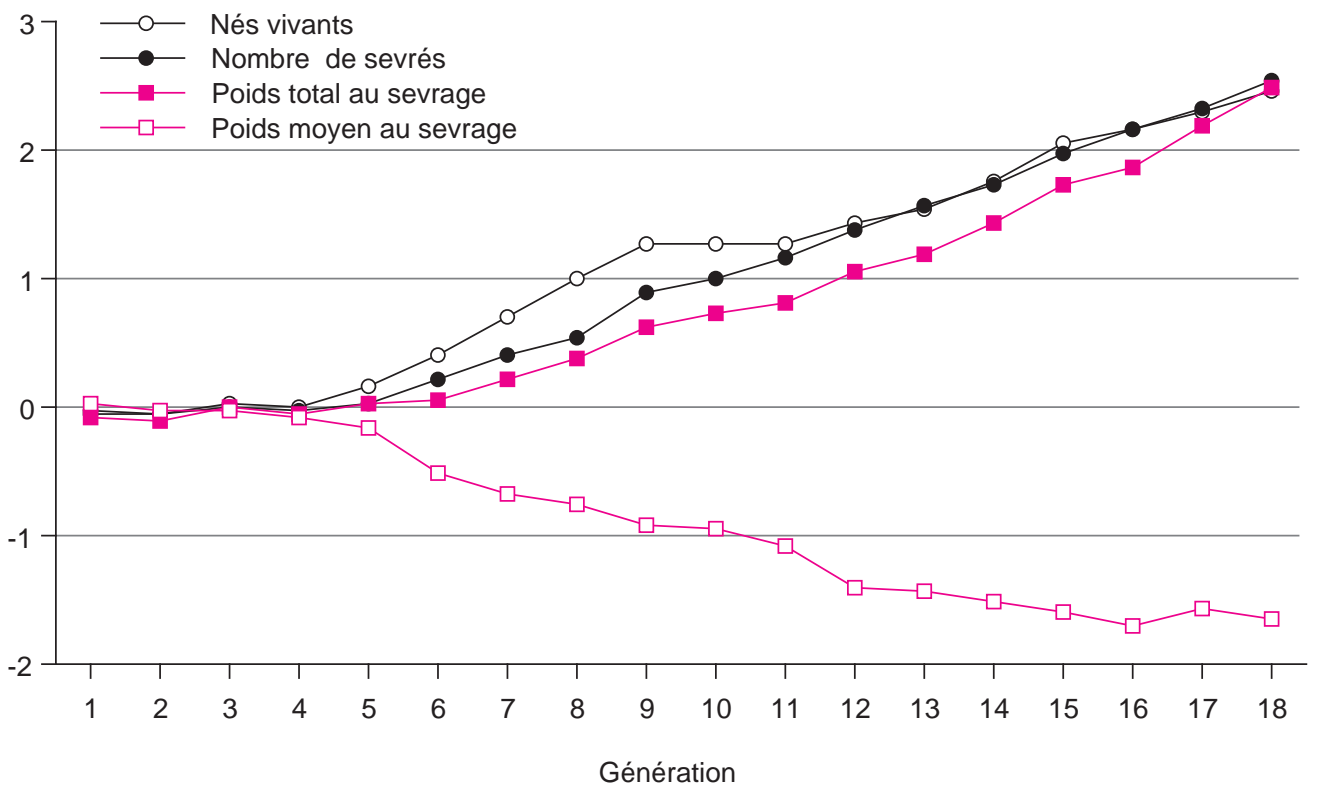

au sevrage a augmenté de $47 \mathrm{~g}$ par génération ; en revanche, le poids individuel moyen au sevrage a diminué de $3,7 \mathrm{~g}$ par génération (figure 2) (Rochambeau 1998).

\section{2 / Essai d'interprétation : influence du surpeuplement utérin sur le poids des lapereaux à la naissance et sur leur croissance}

Nous avons comparé la taille de portée et le poids des lapereaux chez des lapines hémiovariectomisées (dont la corne correspondant à l'ovaire restant est donc surpeuplée) ou " entières " (avec les deux cornes utérines). La réduction de l'espace utérin disponible, à nombre d'implants identique, a un effet défavorable sur la survie fotale et sur le poids à la naissance ; cependant, il est beaucoup plus faible que l'effet défavorable de l'accroissement du nombre total d'embryons (Bolet et al 1996). Ainsi, par exemple, les lapereaux provenant de portées de 8 ou 9 fotus dans une seule corne sont aussi lourds, de la naissance jusqu'à l'âge de 11 semaines, que ceux provenant de portées de 10 ou 11 fotus dans deux cornes. Par conséquent, l'effet défavorable des grandes portées ne semble pratiquement pas dû à un problème de compétition spatiale entre fotus. Il est donc permis de penser que, chez les lapines à forte prolificité, la couverture des besoins de gestation est insuffisante pour permettre une croissance maximale des fœtus. Nous verrons plus loin que la simultanéité de la gestation et de la lactation ne peut qu'amplifier ce problème.

A taille de portée allaitée identique, le nombre de lapereaux nés par portée a un effet sur leur poids individuel jusqu'au sevrage, et même jusqu'à l'âge de 11 semaines. L'écart entre classes extrêmes de taille de portée va en augmentant en valeur absolue, mais en diminuant en valeur relative : il est de l'ordre de $20 \%$ à la naissance (10 à 15 g), $15 \%$ au sevrage (100 à $150 \mathrm{~g})$ et seulement $5 \%$ à 11 semaines (100 à 150 g). Ainsi, les lapereaux ne compensent que partiellement, lors de leur croissance, leur différence de poids à la naissance.

\section{3 / Essai d'interprétation : influence du nombre de lapereaux allaités}

Plusieurs travaux ont été consacrés à l'influence de la taille de portée allaitée sur la croissance des jeunes et les performances de reproduction ultérieures des femelles. Les conclusions sont assez contradictoires (Tudela et al 1998). En fait, il semble que deux effets s'opposent : un effet négatif du nombre de lapereaux allaités par femelle sur leur croissance d'une part, un effet positif du nombre de lapereaux nés sur la production laitière de leur mère, au moins pendant les deux premières semaines, indépendamment $d u$ nombre de lapereaux présents (Bolet et al 1996). Ceci est un argument en faveur de la sélection pour la prolificité avec standardisation des portées à la naissance, bien que la pratique de l'adoption ne permette pas de compenser totalement l'effet défavorable d'une augmentation de la prolificité sur le poids des lapereaux à la naissance.

\section{3 / Longévité et rythme de reproduction}

L'élevage intensif du lapin est caractérisé par une fonte importante du cheptel, entraînant un taux de renouvellement annuel des reproductrices de l'ordre de $120 \%$. Malheureusement, peu de travaux sont consacrés à l'incidence de l'augmentation de la productivité sur la longévité des lapines. Quelques éléments 
permettent cependant de penser que cet effet n'est pas essentiel : la figure 1 met en évidence une augmentation continue de nombre de lapereaux produits par lapine dans leur carrière jusqu'en 1994. Une étude expérimentale (Rinaldo et Bolet 1988) n'a mis en évidence aucune incidence de la valeur génétique des femelles pour la prolificité sur leur longévité.

\section{4 / Compétition gestation-lactation}

\section{1 / Résultats expérimentaux}

Bien que les résultats expérimentaux concernant les effets de la lactation sur le taux d'ovulation et la mortalité embryonnaire soient parfois contradictoires, la fertilité et la prolificité des lapines allaitantes sont globalement plus faibles que celles des lapines non allaitantes. En effet, la lactation a, d'une manière générale, un effet négatif sur le pourcentage de femelles ovulant $(-26 \%)$, le taux de gestation $(-33 \%)$, et la viabilité fotale $(-\square 10 \%)$. De plus, la lactation entraîne une diminution de la croissance pondérale des fœtus $(-20 \%$ à 28 jours de gestation). L'hyperprolactinémie et la faible progestéronémie chez les lapines simultanément gravides et allaitantes, ainsi que le déficit nutritionnel engendré par la production laitière, semblent être les principaux facteurs responsables des effets observés (voir revues de Theau-Clément et Roustan 1992, Fortun-Lamothe et Bolet 1995).

\section{2 / Couverture des besoins nutritionnels de la lapine reproductrice}

Malgré les effets négatifs de la lactation sur les performances de reproduction, la productivité globale d'un élevage (nombre de produits/femelle /unité de temps) est supérieure pour le rythme de reproduction semi-intensif (saillie 11 à 12 jours après la mise bas, environ 47 lapins/femelles/an) comparé au rythme extensif (saillie après le sevrage, environ 34 lapins par femelle et par an). En effet, la diminution de l'intervalle entre mise bas compense la diminution de la taille de la portée à la naissance. Par conséquent, les études des interactions entre la gestation et la lactation chez la lapine doivent être poursuivies. Une approche plus précise des besoins nutritionnels de la lapine simultanément gravide et allaitante à chaque stade de sa vie productive s'avère plus particulièrement nécessaire pour maîtriser le déficit nutritionnel engendré par la lactation et pallier ses inconvénients.

\section{5 / Evolution du mode de conduite}

Depuis quelques années, la conduite des troupeaux de reproductrices évolue, un peu à l'image de l'élevage porcin par le passé. On notera surtout que l'insémination artificielle se développe rapidement, ainsi que la conduite en bandes. Face à ces évolutions, la nécessité de travaux sur la fertilité de la lapine s'est renforcée. En effet, si l'ovulation chez la lapine est provoquée par l'accouplement, ou par une injection d'hormones $(\mathrm{GnRH})$ en insémination artificielle, il n'en reste pas moins vrai que la fertilité des lapines est loin de $100 \%$, soit par refus d'accouplement en saillie naturelle, soit par défaut d'ovulation, de fécondation ou de développement embryonnaire. Des travaux sont actuellement menés sur les facteurs de variation et les posssibilités d'induire l'ovulation, en agissant sur le milieu au sens large (photopériode, injection d'hormones, stress bénéfique, etc., voir la revue de Castellini 1996). La piste de la variabilité génétique de la fertilité n'a pas encore été vraiment explorée.

\section{6 / Evolution des recherches en génétique}

\section{1 / Variabilité génétique de la compétition gestation-lactation}

Les premiers résultats montrent l'importance de la compétition entre lactation et gestation sur le plan nutritionnel. Il semblerait qu'une amélioration de l'alimentation aurait une incidence surtout sur la fonction de lactation et sur les réserves corporelles de la lapine, et moins sur la croissance fœtale (Fortun-Lamothe 1998). Nous nous sommes demandés s'il pouvait y avoir une variabilité génétique de cette " gestion "des besoins simultanés de gestation et de lactation, en relation avec le format des femelles. Une comparaison de l'état des réserves corporelles et des performances de reproduction dans deux souches de femelles différant par leur format adulte a été réalisée. Elle n'a pas permis de mettre en évidence de façon certaine une relation entre format, bilan énergétique et performances de reproduction (Fortun-Lamothe et Bolet 1998).

\section{2 / Prise en compte du poids dans les critères de sélection}

L'amélioration génétique du lapin de chair repose, depuis 1970 , sur l'utilisation d'une femelle métisse de souches prolifiques accouplée à un mâle de croisement terminal apportant une bonne croissance et des qualités bouchères. La recherche de la seule prolificité dans les populations femelles entraîne une diminution du format des souches sélectionnées que ne compense plus le croisement. Le multiplicateur subit un taux d'élimination croissant pour format insuffisant. Le producteur constate une fragilité croissante des reproductrices particulièrement au moment 
de la mise bas, alors que la capacité d'ingestion est limitée chez les femelles légères. Ceci entraîne un déficit alimentaire en début de lactation, déficit qui est aggravé par une portée nombreuse et par le début d'une nouvelle gestation. Pour faire face à la diminution du poids des reproducteurs, un nouveau critère de sélection, le poids du lapereau à l'âge de 63 jours, a été ajouté à l'objectif de sélection de la souche A1077. Cette souche est diffusée; il est donc malaisé d'analyser les conséquences de cette modification de l'objectif sur la fécondité et ses composantes, sur les qualités bouchères et sur l'efficacité alimentaire. Deux expériences de sélection permettront d'analyser cette question et d'entreprendre une étude génétique et biologique des caractéristiques de la croissance ; deux souches de formats initiaux différents seront soumises à une sélection divergente sur le poids à l'âge de 63 jours. Après cinq générations de sélection les évolutions de la fécondité, de l'efficacité alimentaire, des qualités bouchères et des qualités de la viande seront analysées.

\section{Références}

Bolet G., Esparbie J., Falières J., 1996. Relations entre le nombre de fœetus par corne utérine, la taille de portée à la naissance et la croissance pondérale des lapereaux. Ann. Zootech., 45, 185-200.

Castellini C., 1996. Recent advances in rabbit artificial insemination. 6e Congrès mondial de Cuniculture, Toulouse, 9-12 juillet 1996, 2, 13-28.
Fortun-Lamothe L., 1998. Effets de la lactation, du bilan énergétique et du rythme de reproduction sur les performances de reproduction chez la lapine primipare. $7^{\text {e }}$ Journées de la Recherche Cunicole en France, Lyon, 13-14 mai 1998. INRA-ITAVI, Paris, 257-260.

Fortun-Lamothe L, Bolet G., 1995. Les effets de la lactation sur les performances de reproduction chez la lapine. INRA Prod. Anim., 8, 49-56.

Fortun-Lamothe L., Bolet G., 1998. Relations entre le format, l'évolution des réserves corporelles et les performances de reproduction chez la lapine primipare : comparaison de deux types génétiques. 7 es Journées de la Recherche Cunicole en France, Lyon, 13-14 mai 1998. INRA-ITAVI, Paris, 27-30.

Kœhl P.F., 1997. GTE RENALAP 96 : une lapine produit $118 \mathrm{~kg}$ de viande par an. Cuniculture, 138, 247252

Rinaldo D., Bolet G., 1988. Effect of selection for litter size at weaning on reproductive life of female rabbits. $4^{\mathrm{e}}$ Congrès mondial de Cuniculture, Budapest, 10-14 octobre 1988, Genetics and Physiology, 269-275.

Rochambeau H. de, 1998. La femelle parentale issue des souches expérimentales de l'INRA : évolutions génétiques et perspectives. 7 es Journées de la Recherche Cunicole en France, Lyon, 13-14 mai 1998. INRA-ITAVI, Paris, 3-14.

Theau-Clément M., Roustan A., 1992. A study on relationships between receptivity and lactation in the doe and their influence on reproductive performance. J. Appl. Rabbit Res., 15, 412-421.

Tudela F., Poujardieu B., Gauzère J.M., 1998. Productivité de la lapine : préparation des reproducteurs. $7^{\text {es }}$ Journées de la Recherche Cunicole en France, Lyon, 13-14 mai 1998. INRA-ITAVI, Paris, 269-271.

\section{J.-P. BIDANEL}

INRA Station

de Génétique

quantitative

et appliquée

78352 Jouy-en-Josas

Cedex

\section{Conséquences génétiques de l'accroissement de la prolificité des truies}

La généralisation des programmes de sélection " hyperprolifiques » a conduit ces dernières années à une amélioration substantielle de la taille de la portée à la naissance dans les élevages de sélection et de multiplication. Les effets de cette sélection sont désormais perceptibles au niveau des élevages de production. Cet accroissement de la prolificité des truies va vraisemblablement s'amplifier dans les années à venir, lorsque les effets liés à la mise en place d'une évaluation génétique de type BLUP-modèle animal et à l'introduction des lignées synthétiques sino-européennes ou des races chinoises dans les schémas de croisement se feront sentir. Ce texte fait le point sur les conséquences génétiques de cette augmentation de la taille de la portée à la naissance à partir des résultats expérimentaux et des estimations de paramètres génétiques disponibles dans la littérature.

\section{1 / Conséquences génétiques d'une sélection sur la prolificité}

\section{1 / Sur les performances de reproduction}

La sélection pour la prolificité est le plus souvent basée sur le nombre de porcelets nés totaux (NT) par portée. Ce choix n'est pas 